\title{
Deficient histone acetylation and excessive deacetylase activity as epigenomic marks of prostate cancer cells
}

\author{
SHUNDONG CANG ${ }^{1,2^{*}}$, JINGYANG FENG ${ }^{2 *}$, SENSUKE KONNO ${ }^{3}$, LIYING HAN ${ }^{4}$, KATHERINE LIU ${ }^{2}$, \\ SANSAR C. SHARMA ${ }^{5}$, MUHAMMAD CHOUDHURY ${ }^{3}$ and J.W. CHIAO ${ }^{2}$ \\ ${ }^{1}$ Department of Oncology, The First Affiliated Hospital, Zhengzhou University, Zhengzhou, \\ Henan 450052, P.R. China; Departments of ${ }^{2}$ Medicine, ${ }^{3}$ Urology, ${ }^{4}$ Pathology and \\ ${ }^{5}$ Ophthalmology, New York Medical College, Valhalla, NY 10595, USA
}

Received April 7, 2009; Accepted May 27, 2009

DOI: 10.3892/ijo_00000459

\begin{abstract}
Aberrant epigenomic alterations include incorrect histone modifications involving altered expression of chromatin-modifying proteins. They contribute to gene silencing and carcinogenesis. The nature of the epigenomic alterations occurring with prostate cancer remains to be fully identified. The acetylation status of histone $\mathrm{H} 3$ in human prostate cancer cells was assessed with multiple acetylation sites at $\mathrm{N}$-termini. In contrast to the non-malignant prostatic cell lines $\mathrm{RC} 165 \mathrm{~N} / \mathrm{h}$ and $\mathrm{RC} 170 \mathrm{~N} / \mathrm{h}$ which possess stem cell properties, cancer cell lines LNCaP, DU-145, and PC-3 were either not acetylated or reduced in density (50-70\%), at $\mathrm{N}$-termini lysines 9, 14, 18, and 23 of histone H3. Deficient acetylation of histone $\mathrm{H} 3$ was similarly detected with clinical prostatic adenocarcinomas as compared to normal tissues. Cancer cell lines and adenocarcinomas exhibited varied acetylation status at particular lysines, indicating the possible presence of deacetylation patterns reflecting individual cancer cell clones. A significantly elevated activity of histone deacetylases (HDACs) was determined in both cancer cell lines and adenocarcinomas. Inhibition of HDACs enhanced histone acetylation and p21 gene expression, indicating that excessive HDAC activity is a requisite for deficient histone acetylation. Deficient histone acetylation involving excessive HDAC activity may represent epigenomic features of prostate cancer cells, and the aberrant enzyme activity is probably an underlying cause of disrupting the epigenomes of normal prostatic cells.
\end{abstract}

Correspondence to: Dr J.W. Chiao, Department of Medicine, New York Medical College, Valhalla, NY 10595, USA

E-mail: jen-wei_chiao@nymc.edu

${ }^{*}$ Contributed equally

Key words: epigenetics, histone acetylation, deacetylation, histone deacetylase, prostate cancer

\section{Introduction}

Two copies of each histone protein $\mathrm{H} 2 \mathrm{~A}, \mathrm{H} 2 \mathrm{~B}, \mathrm{H} 3$, and $\mathrm{H} 4$ form an octamer core of nucleosome, wrapped around by 1.65 turns of DNA. Each form of histone has variants as some amino acids are enzymatically modified. The posttranslational modifications include methylation, acetylation, phosphorylation, ubiquitylation, and others that may alter the structural properties of the protein, as well as the chromatin architecture that may be transmissible to the next generations as epigenetic inheritance. The various types of histone modifications and their combinations are considered the specific instructions for the chromatin functions and nuclear processes $(1,2)$. Most of the histone modifications occur around the flexible $\mathrm{N}$ - and $\mathrm{C}$-termini. The $\mathrm{N}$-termini are highly basic, enriched with amino acids that could be acetylated and methylated. The N-terminal loops are intertwined with the DNA, passing through the channels in the superhelix and protruding from the surface of nucleosomes (3). The different types of histone modifications have been linked with distinct functions. Acetylation has been known to alter the structural property of nucleosomes and chromatin, enhancing gene expression as well as DNA repair and cytokine-activated signal transduction (4-6). The lysine acetylation marks may be interpreted by interacting with specific effector modules bromodomain complexes $(7,8)$, which may mediate chromatin remodeling and transcriptional regulation.

The level of acetylation is maintained by a pair of opposing enzymes, the acetyltransferases and the histone deacetylases (HDACs). Aberrant activities of these enzymes such as excessive recruitment of HDACs, and mutation in acetyltransferases, have been implicated in gene silencing in cancer (9-11). There have been cancer clinical trials employing HDAC inhibitors, but the scientific rationale for treating prostate cancer has not been fully presented. Our recent experiments using the isothiocyanates as HDAC inhibitors, in prostate cancer cells, revealed that they induced hyperacetylation of histones $\mathrm{H} 3$ and $\mathrm{H} 4$, resulting in growth arrest and apoptosis $(12,13)$. The results elucidated the presence of aberrant histone modifications in the prostate cancer cells. We have 
A

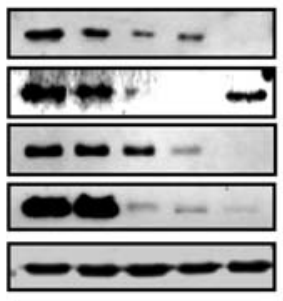

$\mathrm{Ac} \mathrm{H} 3 \mathrm{~K} 9$

Ac H3K14

Ac H3K18

$\mathrm{Ac} \mathrm{H} 3 \mathrm{~K} 23$

Histone $\mathrm{H} 3$

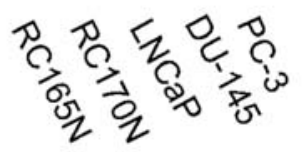

B

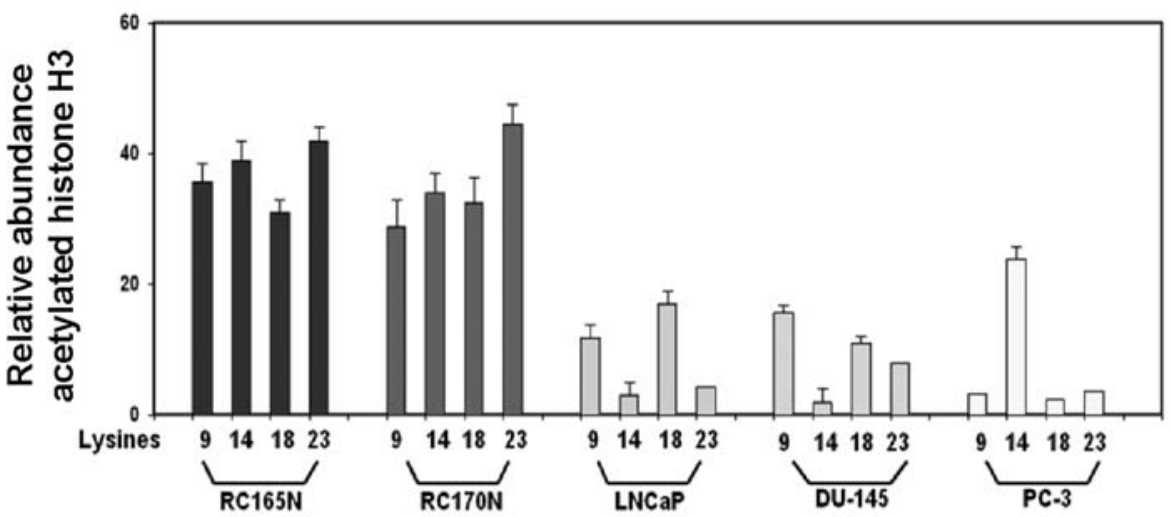

Figure 1. Deficient acetylation of histone H3 N-terminal lysines in prostate cancer cell lines. (A) Status of acetylation of N-terminal lysines 9, 14, 18, and 23 of histone $\mathrm{H} 3$ in non-malignant prostatic cell lines with stem cell properties (RC165N/h, RC170N/h), and prostate cancer lines (LNCaP, DU-145, PC-3) was determined using histone preparations by Western blotting with infrared detection and analyses. Antibody against histone H3 was used as a loading control. (B) Histograms depicting relative abundance of acetylated lysines 9, 14, 18, and 23 of histone H3 of each cell line. The histograms represent the mean expression levels (arbitrary unit) of three Western blot determinations of acetylated histone H3 lysines of each cell line, relative to the quantity of histone $\mathrm{H} 3 \pm \mathrm{SD}$.

since focused our analyses on histone $\mathrm{H} 3$ of prostate cancer cells, because the majority of acetylation and methylation occur here (14). We examined the lysine residues, known to be acetylated in vivo, located from the N-terminus extending near the channel in the seperhelix where the histone protrudes to the surface of nucleosome (15). In this study we present the profiles of histone $\mathrm{H} 3 \mathrm{~N}$-terminal acetylation in prostate cancer cell lines and in clinical adenocarcinomas. They show a severe deficiency of acetylation which is distinct from the profile of non-malignant prostatic cells. The deficient acetylation is caused by excessive HDAC activity and could be reversed by HDAC inhibition.

\section{Materials and methods}

Cell culture and tissues. Prostate cancer cell lines LNCaP, DU-145, and PC-3 were maintained in RPMI-1640 with $10 \%$ heat-inactivated fetal calf serum. Some of these cultures were exposed to $2 \mathrm{mM}$ sodium butyrate for one day. Two telomerase-immortalized non-malignant human prostate epithelial cells, RC165N/h and RC170N/hclone 7 (RC170N/h) that retain multipotent stem cell properties $(16,17)$, were kindly provided by Dr Johng S. Rhim, Uniformed Services University of the Health Sciences, Bethesda, MD. They were maintained in serum-free keratinocyte medium supplemented with bovine pituitary extract and recombinant epidermal growth factor (Life Technologies, Inc.). Prostate specimens were from discarded tissues after pathological examinations according to the internal review board protocol. The adenocarcinoma specimens were obtained from radical prostatectomy and the tumors reported were determined pathologically with the following grades and stages. Specimens 1-6, presented in the report were respectively moderately differentiated (Gleason score 6 , stage $\mathrm{T}_{0} \mathrm{~N}_{0}$ ); moderately differentiated (Gleason score 6 , stage $\mathrm{T}_{2 \mathrm{~b}}$ ); poorly differentiated (Gleason score 6-7, stage $\mathrm{T}_{2 \mathrm{~b}}$ ); moderately differentiated (Gleason score 5, stage $\mathrm{T}_{2 \mathrm{~b}}$ ); moderate to poorly differentiated (Gleason score 6, stage $\mathrm{T}_{2 \mathrm{c}}$ ); and moderate to poorly differentiated (Gleason score 7 , stage $\mathrm{T}_{2 \mathrm{c}}$ ). Two prostatic tissues (normal) were determined pathologically as being non-cancerous, without hypertrophy, and no diagnostically abnormality.

Histone modification and detection. Lysates from cell cultures or tissues were prepared in the presence of HDAC inhibitor sodium butyrate at $5 \mathrm{mM}$ final concentration as previously described. Histones were isolated using an acid extraction procedure $(18,19)$. The procedures for quantitative Western blotting were performed as previously described (18), with site-specific antibodies against acetylated lysines 4, 9, 14, 23, or 27 of histone H3 (Millipore). For confocal immuno- 
fluorescence microscopy, DU-145 cells grown on chamber slides were treated one day without or with $2 \mathrm{mM}$ sodium butyrate, then fixed, permiabilized, blocked in BSA and incubated with a rabbit anti-histone $\mathrm{H} 3$ for $1 \mathrm{~h}$. A fluoreceinconjugated $\mathrm{F}\left(\mathrm{ab} \mathrm{b}^{\prime}\right)_{2}$ fragment of goat anti-rabbit Ig was used as a second antibody and the DNA counterstained with propidium iodide (PI) to visualize the nuclei of all cells. Images were captured using an MRC 1024 ES confocal laser scanning microscopy system. Identical image settings were used for experimental conditions.

HDAC activity. HDAC activity of classes I, II, and Sirt1 was detected with a previously described procedure using a HDAC assay kit (Biomol International) (18). The assay uses a synthetic acetylated lysine side chain as a substrate to react with the deacetylase samples in a 96-well microplate. Deacetylation of the substrate allows its detection with a developer that produces a fluorophore. A HeLa cell nuclear extract with known HDAC activity was used as a positive control. Some samples were added with $1 \mu \mathrm{M}$ trichostatin A (TSA), a known HDAC inhibitor. The reaction mixture was added with the substrate with concentrations at or below the $\mathrm{Km}$, and incubated at room temperature before adding the developer containing $1 \mu \mathrm{M}$ TSA. The reaction was determined with a Cary Eclipse fluorimeter using wavelength $360 \mathrm{~nm}$ and detection of emitted light at $460 \mathrm{~nm}$. The fluorescence was expressed as arbitrary units (AU). The mean values of fluorescence for HDAC activity from the malignant and non-malignant cells were statistically compared with the two-tailed Student's t-test, and a $\mathrm{P}<0.05$ was considered to be statistically significant.

\section{Results}

Deficient histone acetylation in prostate cancer cells. The status of acetylation of histone $\mathrm{H} 3$ from three prostate cancer cell lines, LNCaP, DU-145 and PC-3, was compared to two non-malignant prostatic cell lines RC165N/h and RC170N/h, which were cloned from telomerase-immortalized primary non-malignant prostate tissues. They express embryonic stem cell marker Oct-4, prostate stem cell markers CD133, CD44 and retain the properties of multipotent stem cells including differentiating into multiple prostatic tissues (16). Acetylation levels of the N-terminal lysines 9, 14, 18, or 23 of histone H3 were examined by Western blotting. Fig. 1 depicted that cells from the two non-malignant cell lines were acetylated, with similar levels at the N-terminal lysines. In contrast, the acetylation density of the three cancer cell lines was significantly lower, at each of these lysines. There were clear variations of acetylation levels at particular lysine sites, among the cancer cell lines. The LNCaP and DU-145 cells were more similar, comparing to the PC-3 cells. They lack acetylation at lysine 14, and the levels of acetylated lysines 9,18 , and 23 were about one third of those in the nonmalignant cells (Fig. 1). The PC-3 cells were clearly acetylated at lysine 14, and acetylation on lysines 9, 18 and 23 was barely detectable. The results demonstrated distinct patterns of histone acetylation between prostate cancer and nonmalignant cell lines, with the cancer cells either reduced, or absent in the N-terminal acetylation.
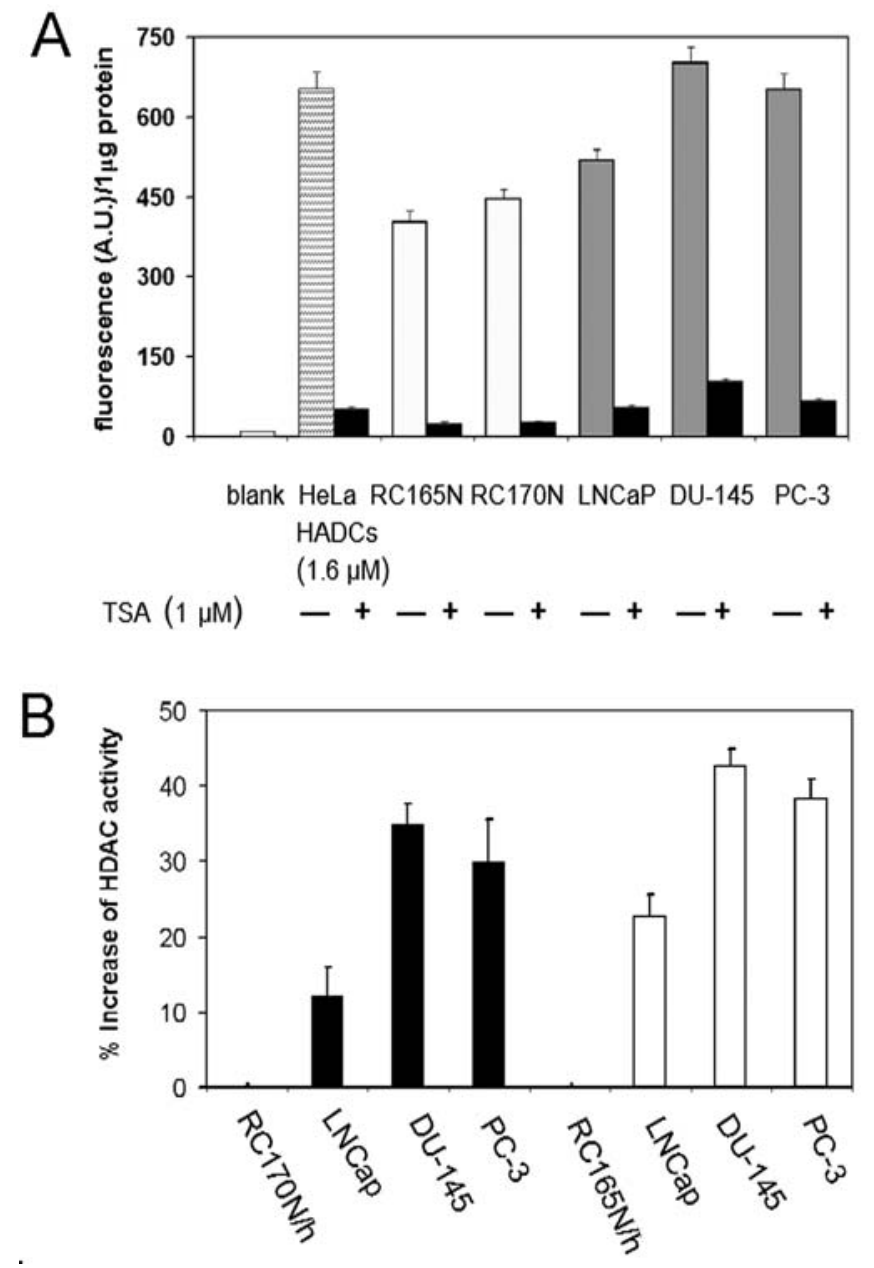

Figure 2. Elevated levels of the activity of HDACs in prostate cancer cell lines. (A) The graph depicts the activity of HDACs, in arbitrary unit of fluorescence, is elevated in the three cancer cell lines (LNCaP, DU-145, and PC-3) comparing to the two non-malignant cell lines (RC165N, RC179N), assayed with equal lysate proteins. (ם) indicates the levels of HDAC activity after treated with TSA. Data points represent means \pm SD from three experiments. The difference in fluorescence between the cancer and nonmalignant cells is statistically significant $(\mathrm{P}<0.05)$. (B) The graph shows $\%$ increase of the activity of HDACs in the three cancer cell lines ( $\mathrm{LNCaP}$, DU-145, PC-3) comparing to either RC170N/h (left side of graph), or $\mathrm{RC} 165 \mathrm{~N} / \mathrm{h}$ (right side of the graph).

Excessive HDAC activity and the effects of HDAC inhibition. A deficient histone acetylation would imply an excessive deacetylation, which could be caused by an elevated activity of the deacetylase enzymes. The total HDAC activity of classes I, II, and Sirt1 from each cell culture was quantified, and Fig. 2A depicted clearly that all three cancer cell lines exhibited higher HDAC activity, per unit amount of cellular protein, compared to the non-malignant cell lines. The difference of HDAC activity between $\mathrm{RC} 165 \mathrm{~N} / \mathrm{h}$ and $\mathrm{RC} 170 \mathrm{~N} / \mathrm{h}$ was not statistically significant $(\mathrm{P}=0.08)$, while the HDAC activity in the cancer cells LNCaP, DU-145, or PC-3 was significantly higher than either $\mathrm{RC} 165 \mathrm{~N} / \mathrm{h}$ or $\mathrm{RC} 170 \mathrm{~N} / \mathrm{h}(\mathrm{P}<0.05)$. When the cellular proteins were treated with $1 \mu \mathrm{M}$ TSA, an inhibitor of HDACs, the great majority of the HDAC activity was blocked. These results indicated a higher HDAC activity in the prostate cancer cells. Fig. 2B shows that the LNCaP cells had $\sim 12-23 \%$ higher activity than the two non-malignant cells, the DU-145 cells were $35-42 \%$ higher, and the PC-3 cells $30-38 \%$ higher. 
A
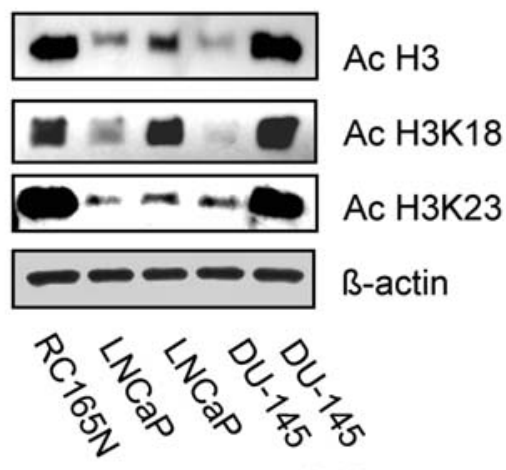

$--++\mathrm{NaBt}$

(2 mM)

C

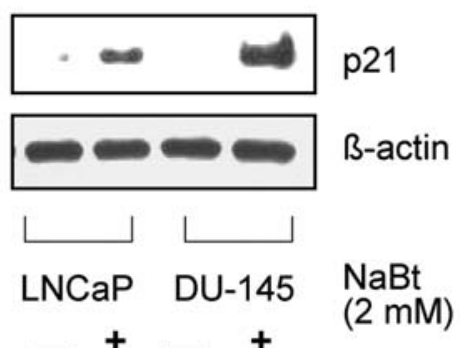

B $\mathrm{RC} 165 \mathrm{~N} / \mathrm{h} \quad \mathrm{DU}-145 \quad \mathrm{DU}-145+\mathrm{NaBt}$

$\mathrm{Ac} \mathrm{H} 3$
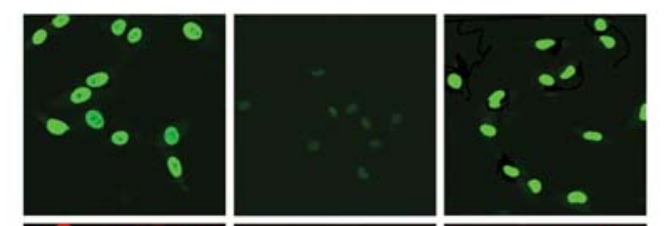

$\mathrm{PI}$
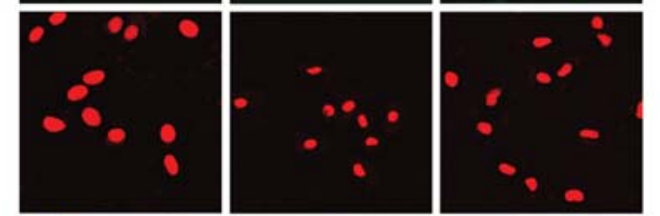

Merge

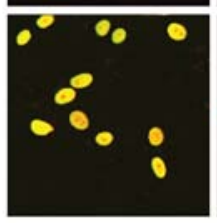

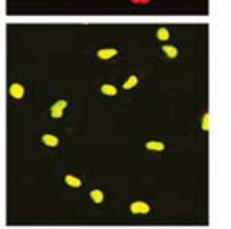

Figure 3. Reversal of deficient acetylation of histone H3 by inhibiting HDACs. (A) Status of acetylated lysines of histone H3 of cancer cell lines LNCaP and DU-145, shown by Western blotting, with or without treated with HDAC inhibitor sodium butyrate. Treated cells show an enhancement of lysine acetylation, with some levels comparable to the non-malignant cells RC165N/h. (B) Cancer cells DU-145 without or with sodium butyrate treatment were examined for the expression of acetylated histone $\mathrm{H} 3$, using fluorescent microscopy with confocal analyses, magnification x200, oil, Zeiss. The status of acetylated histone $\mathrm{H} 3$ of non-malignant cells $\mathrm{RC} 165 \mathrm{~N} / \mathrm{h}$, not treated with sodium butyrate was used as a comparison. PI was used to identify all nuclei for the images. (C) Western blot shows enhancement of p21 level, in LNCaP and DU-145 cell lysates after sodium butyrate treatment. Antibody against 3 -actin was used as a loading control.

To further determine that the lower level of histone acetylation is related to an excessive deacetylase activity, the status of acetylation was examined after the cancer cells were treated with sodium butyrate, an inhibitor of HDACs. Fig. 3A shows that the acetylation levels of histone $\mathrm{H} 3$, and those at lysines 18 and 23 in LNCaP and DU-145 cells, were clearly increased after sodium butyrate treatment, when compared to that of non-malignant cells $\mathrm{RC} 165 \mathrm{~N} / \mathrm{h}$. When analyzed with confocal fluorescent microscopy (Fig. 3B), DU-145 had background level of acetylated histone $\mathrm{H} 3$ in the nuclei. The sodium butyrate treatment enhanced fluorescent staining to the level of the non-malignant cells RC165N/h. These findings verified the results that an aberrantly elevated HDAC activity may be responsible for the deficient acetylation in prostate cancer cells.

It has been reported that highly acetylated histones, as induced by HDAC inhibitors, are associated with the activation of the p21 gene, inhibitor of cyclin-dependent kinases $(20,21)$. The expression level of p21 in the prostate cancer cell lines, without or with the treatment of sodium butyrate was examined. Fig. 3B shows convincingly that as the result of sodium butyrate treatment, the protein levels of p21 were significantly up-regulated, in both LNCaP and DU-145 cells.

The status of histone $\mathrm{H} 3$ acetylation was examined in eleven prostate adenocarcinoma specimens, and compared to four normal prostate specimens. Acetylation at multiple sites of histone $\mathrm{H} 3$ was severely deficient in all the adenocarcinomas examined, and representative data are described in Fig. 4A. Comparing to the normal specimens, acetylation density of histone $\mathrm{H} 3$ from the adenocarcinomas was generally weak, and were absent at lysines 14 and 23 for most of the specimens. There were some variations of the deficiency among the adenocarcinomas. For example, specimens 1 and 6 lost acetylation at lysine 9 as compared to that of specimens 2, 3, and 4. Fig. 4B illustrates the HDAC activity of these prostate specimens as measured per unit of cellular proteins. Each adenocarcinoma specimen had higher activity than the normal specimens, with the difference statistically significant $(\mathrm{P}<0.02)$. The results indicate that the HDAC enzyme activity may be elevated in the prostatic adenocarcinomas.

\section{Discussion}

Evidence is presented in this report that prostate cancer cells have either lost acetylation completely or are reduced in levels, at multiple acetylation sites of histone H3 N-termini, revealing a deficient state of histone acetylation. For each analysis, four consecutive lysines starting from the $\mathrm{N}$-termini were examined for the status of histone acetylation. This method generated more complete profiles of acetylation 
A

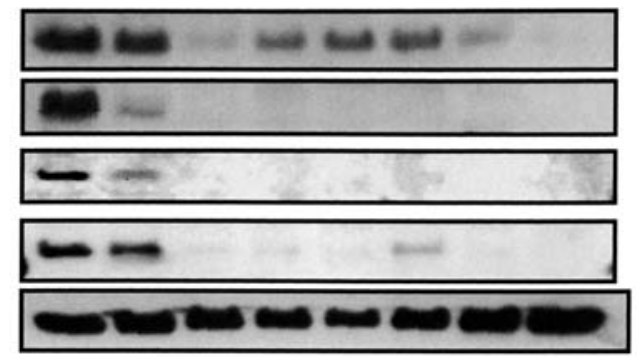

Ac H3K9

Ac H3K14

Ac H3K18

Ac H3K23

ß-actin

B
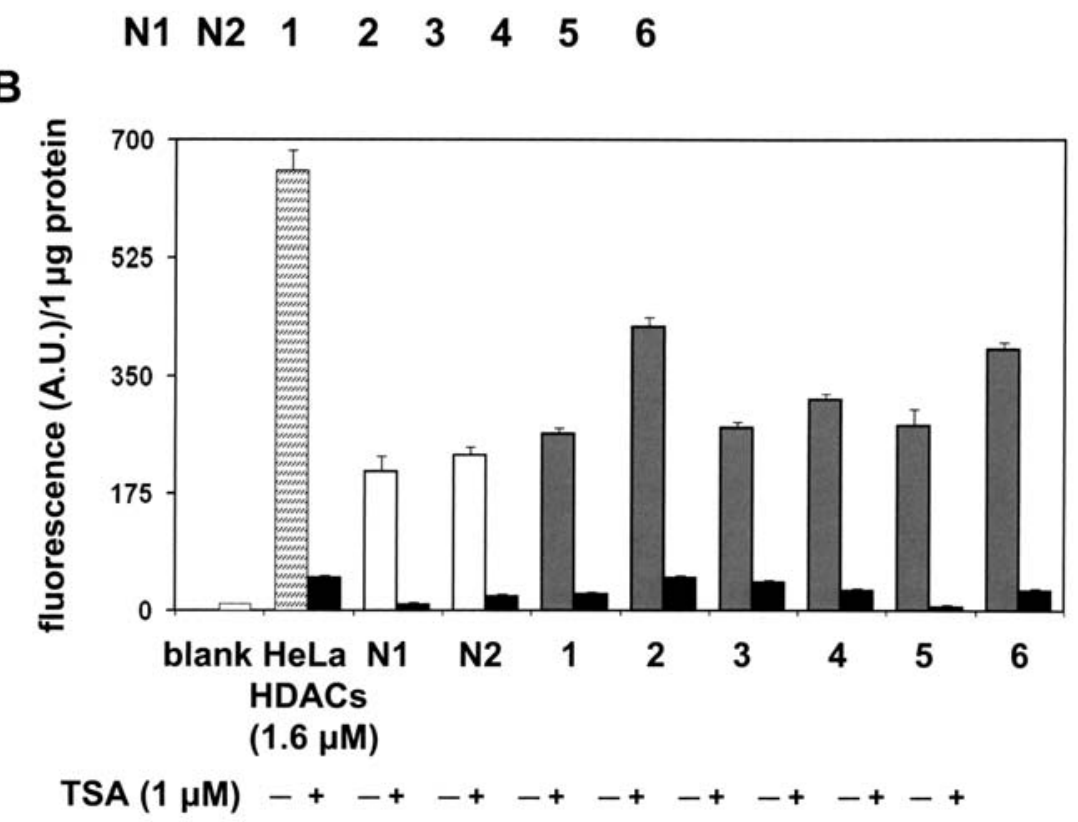

Figure 4. Deficient acetylation of histone H3 N-terminal lysines and elevated activity of HDACs in clinical prostatic adenocarcinomas. (A) Western blots show either reduced or absent expression of acetylated lysines 9, 14, 18, and 23 of histone $\mathrm{H} 3$ in the tissue lysates of prostatic adenocarcinomas (1, 2, 3, 4, 5, and 6) as compared to the normal prostatic tissues (N1 and N2). (B) The graph depicts the activity of HDACs, in arbitrary unit of fluorescence, is elevated in adenocarcinomas (1, 2, 3, 4, 5, and 6) comparing to the normal tissues (N1 and N2), assayed with equal lysate proteins. ( $\mathbf{\square})$ indicates the levels of fluorescence after TSA treatment. Data points represent means \pm SD from three separate experiments. The difference in fluorescence between each adenocarcinoma and either $\mathrm{N} 1$ or $\mathrm{N} 2$ normal tissue is statistically significant $(\mathrm{P}<0.05)$.

than examining single lysines. The experimental results have clearly demonstrated the presence of a deficient histone acetylation along with an excessive deacetylase activity in both prostate cancer cell lines and clinical adenocarcinomas, as compared to non-malignant prostatic cell lines and tissues. The non-malignant prostatic cell lines, used for comparative analysis, retain embryonic stem cell markers and multipotent stem cell properties $(16,17)$. Whether the incorrect histone acetylation in the cancer cells implies a disruption of the normal stem cell epigenomes remains to be determined.

One potential mechanism for initiating deficient histone acetylation, and establishing the marks as irreversible epigenomic changes, could be that the expression of the chromatin-modifying proteins and enzymes, which maintain the balance of histone acetylation, become abnormal. This possibility was examined with the HDAC activity, which was determined to be elevated with statistical significance, in both cancer cell lines and adenocarcinomas examined. Additionally, the low level histone acetylation could be consistently up-regulated after HDAC inhibition, further supporting an excessive deacetylase activity. This finding is significant since there is no prior report clearly defining an elevated HDAC activity in clinical prostate tumors. Since the HDAC activity measured in this study represented the sum of multiple HDAC species, this analysis therefore could not decipher the activity levels of individual enzymes. How the activity levels of individual HDACs affect the $\mathrm{N}$-terminal lysines or global acetylation, remains to be investigated. This will shed light on the difference seen between the magnitude of increased HDAC activity and the decreased acetylation at the $\mathrm{N}$-termini of histone $\mathrm{H} 3$. The current findings are in line with other reports. Patra et al (22) described that HDAC activity was 2- to 3-fold higher in prostate cell lines TSUPr1 and DuPro, than a benign prostatic hyperplasia cell line BPH-1, but the effect on histone acetylation was not described. Seligson et al (23) described a potential association of a lower density of histone acetylation at H3K9 or H3K18 with certain grades of prostate adenocarcinomas, but no measurement was made with enzymes regulating acetylation. An excessive recruitment of HDAC activity was also described with other cancers including leukemic cells (24).

The experimental results demonstrated that the prostate cancer cell lines may have individual patterns of histone 
deacetylation, i.e., lost or reduced acetylation at particular lysine residues. The LNCaP and DU-145 cell lines were shown to have a similar pattern that is distinct from the PC-3 cells. Whether the individual deacetylation profiles represent the aberrant acetylation marks of the original clones, transmitted as epigenetic inheritance, or reflect those deacetylation marks acquired during tumor progression, remains to be analyzed. The observations imply that each pattern of deacetylation may represent an epigenetic mark of the cell type. The combination of the status of histone acetylation and the activity level of HDACs could be yet another characteristic as a cell marker. The current initial analyses have established the methodology used, i.e., examining multiple acetylation sites in combination with HDAC activity, which could be employed for further analyses of the cells of adenocarcinomas and pre-cancerous lesions. The new information may further reveal any association of tumor developmental stages with histone deacetylation profiles of cancer cells.

The experimental results imply the presence of dysregulated epigenomes in prostate cancer cells, which include an excessive HDAC activity and deficient histone acetylation. Since HDACs are present in the complexes of transcriptional co-regulators such as mSin $3 \mathrm{~A}$ and $\mathrm{N}-\mathrm{CoR}$ which are required for repressing target genes $(25,26)$, the use of HDAC inhibitors could de-repress the genes that are inactivated due to hypoacetylation. Reactivation of the cell cycle regulator p21 gene in prostate cancer cells, after inhibiting HDACs, may represent an example in this regard. These findings point to the potential of HDAC inhibitors to restoring histone acetylation and their functions to a more normal state.

\section{Acknowledgements}

S.C. is a CAHON Research Scholar (CAHON.org) and recipient of a fellowship grant from the International Scholar Exchange Foundation.

\section{References}

1. Strahl BD and Allis CD: The language of covalent histone modifications. Nature 403: 41-45, 2000.

2. Turner BM: Histone acetylation and an epigenetic code. Bioessays 22: 836-845, 2000

3. Luger K, Mader AW, Richmond RK, Sargent DF and Richmond TJ: Crystal structure of the nucleosome core particle at 2.8 A resolution. Nature 389: 251-260, 1997.

4. Taunton J, Hassig CA and Schreiber SL: A mammalian histone deacetylase related to the yeast transcriptional regulator Rpd3p. Science 272: 408-411, 1996.

5. Kim SC, Sprung R and Chen Y: Substrate and functional diversity of lysine acetylation revealed by a proteomics survey. Mol Cel 23: 607-618, 2006.

6. Yang XJ and Gregoire S: Metabolism, cytoskeleton and cellular signaling in the grip of protein Nepsilon- and O-acetylation. EMBO Rep 8: 556-562, 2007.

7. Dhalluin C, Carlson JE and Zeng L: Structure and ligand of a histone acetyltransferase bromodomain. Nature 399: 491-496, 1999.
8. Paro R and Hogness DS: The Polycomb protein shares a homologous domain with a heterochromatin-associated protein of Drosophila. Proc Natl Acad Sci USA 88: 263-267, 1991.

9. Marks PA, Richon VM and Rifkind RA: Histone deacetylases inhibitors: inducers of differentiation or apoptosis of transformed cells. J Natl Cancer Inst 92: 1210-1216, 2000.

10. Brehm A, Nielsen SJ, Miska EA, McCance DJ, Reid JL, Banniste AJ and Kouzarides T: The E7 oncoprotein associates with Mi2 and histone deacetylase activity to promote cell growth. EMBO J 18: 2449-2458, 1999

11. Gayther SA, Batley SJ, Linger L, et al: Mutations truncating the EP300 acetylase in human cancers. Nat Genet 24: 300-303, 2000.

12. Wang LG, Liu XM, Fang Y, et al: De-repression of the p21 promoter in prostate cancer cells by an isothiocyanate via inhibition of HDACs and c-Myc. Int J Oncol 33: 375-380, 2008.

13. Beklemisheva AA, Fang Y, Feng J, Ma X, Dai W and Chiao JW: Epigenetic mechanism of growth inhibition induced by phenelhexyl isothiocyanate in prostate cancer cells. Anticancer Res 26: 1225-1230, 2006.

14. Felsenfeld $\mathrm{G}$ and Groudine M: Controlling the double helix. Nature 421: 448-453, 2003.

15. Latham JA and Dent SY: Cross-regulation of histone modifications. Nat Structl Mol Biol 14: 1017-1024, 2007.

16. Li H, Zhou J, Miki J, Furusato B, Gu Y, Srivastava S, McLeod DG, Vogel JC and Rhim JS: Telomerase-immortalized non-malignant human prostate epithelial cells retain the properties of multipotent stem cells. Exp Cell Res 314: 92-102, 2008.

17. Gu Y, Li H, Miki J, Kim KH, Furusato B, Sesterhenn IA, Chu WS, McLeod DG, Srivastava S, Ewing CM, Isaacs WB and Rhim JS: Phenotypic characterization of telomeraseimmortalized primary non-malignant and malignant tumorderived human prostate epithelial cell lines. Exp Cell Res 312: 831-843, 2006.

18. Wang LG, Beklemisheva A, Liu XM, Ferrari AC, Feng J and Chiao JW: Dual actions on promoter demethylation and chromatin by an isothiocyanate restored GSTP1 silenced in prostate cancer. Mol Carcinog 46: 24-31, 2007.

19. Yoshida M, Kijima M, Akita M and Beppu T: Potent and specific inhibition of mammalian histone deacetylase both in vivo and in vitro by trichostatin A. J Biol Chem 265: 17174-17179, 1990.

20. Lu Q, Lin X, Feng J, Zhao X, Gallagher R, Lee MY, Chiao JW and Liu D: Phenylhexyl isothiocyanate has dual function as histone deacetylase inhibitor and hypomethylating agent and can inhibit myeloma cell growth by targeting critical pathways. J Hematol Oncol 1: 6-15, 2008.

21. Gui CY, Ngo L, Xu WS, Richon VM and Marks PA: Histone deacetylase (HDAC) inhibitor activation of p21 $1^{\mathrm{WAF}}$ involves changes in promoter-associated proteins, including HDAC1. Proc Natl Acad Sci USA 101: 1241-1246, 2004.

22. Patra SK, Patra A and Dahiya R: Histone deacetylase and DNA methyltransferase in human prostate cancer. Biochem Biophys Res Commun 287: 705-713, 2001.

23. Seligson DB, Horvath S, Shi T, Yu H, Tze S, Grunstein M and Kurdistani SK: Global histone modification patterns predict risk of prostate cancer recurrence. Nature 435: 1162-1166, 2005.

24. Lin RJ, Nagy L, Inoue S, Shao W, Miller WH Jr and Evans RM: Role of the histone deacetylase complex in acute promyelocytic leukaemia. Nature 391: 811-814, 1998.

25. Hildebrand D, Tiefenbach J, Heinzel T, Grez M and Maurer AB: Multiple regions of ETO cooperate in transcriptional repression. J Biol Chem 276: 9889-9895, 2001.

26. Minucci S, Nervi C, Coco FL and Pelicci PG: Histone deacetylases: a common molecular target for differentiation treatment of acute myeloid leukemias? Oncogene 20: 3110-3115, 2001. 